\title{
DEUX LETTRES DE MARTIN JUGIE À SERGE BOULGAKOV
}

\author{
Job Getcha et Vassa Kontouma-Conticello
}

Théologien et «historien des Églises séparées » ${ }^{1}$, fidèle contributeur des Échos d'Orient et du Dictionnaire de Théologie catholique, le père assomptionniste Martin Jugie (1878-1954) est principalement connu des byzantinistes pour ses nombreux articles érudits, ses éditions de textes et sa monumentale Theologia dogmatica Christianorum Orientalium (Paris 1926-1936) ${ }^{2}$. Si l'on s'est parfois arrêté sur le ton très polémique de certains de ses écrits ${ }^{3}$, on a encore très peu considéré son point de vue personnel sur l'union des Églises orientales avec l'Église catholique romaine, dans cette période située entre les Conciles de Vatican I (1869-1870) et de Vatican II (1962-1965).

Or, tout au long de sa vie, Jugie semble avoir été d'une grande constance dans sa représentation de l'Église universelle et dans son approche des Orientaux, en particulier des orthodoxes ou «Gréco-Russes », selon sa propre terminologie ${ }^{4}$. Ainsi, La prière pour l'unité chrétienne (Paris 1920), enthousiaste œuvre de ses débuts ${ }^{5}$, pose distinctement les principes de la synthèse finale que constitue Où se trouve le christianisme intégral (Paris 1947) et qu'il avait préparée, selon la méthode historico-doctrinale qui lui est chère, dans Le Schisme byzantin (Paris 1941) ${ }^{6}$. L'Église, pour Jugie, est visible et

1. V. Laurent, L'œuvre scientifique du R. P. Martin Jugie, REB 11, 1953, p. 7.

2. Ibidem, p. 19-32 (bibliographie complète).

3. Ibidem, p. 17. On remarquera seulement ici que le ton particulier adopté par Jugie dans nombre de ses écrits trouve son inspiration dans la langue et la pensée de Joseph de Maistre (1753-1821). Voir M. Jugie, Joseph de Maistre et l'Église gréco-russe, Paris 1922.

4. Nommer l'orthodoxie est pour Jugie une question centrale, à laquelle il a consacré deux publications : Joseph de Maistre et le schisme gréco-russe. II, Dénominations et caractères généraux des Églises dissidentes issues du schisme, EO 20, 1921, p. 414-430; Les divers noms de l’Église gréco-russe, Bessarione 37, 1922, p. 179-199. Cf. V. Conticello, Nommer l'orthodoxie, Annuaire de l'EPHE-SR 109, 2000-2001, p. 371.

5. L'engagement de l'auteur pour la cause de l'union y apparaît comme total. Voir ainsi Prière, p. 237: «Au lieu d'un souvenir dans ses prières, de quelques actes de vertu offerts à cette intention, on peut consacrer sa vie entière à travailler au rétablissement de l'unité chrétienne, faire converger vers ce but toute son activité spirituelle, s'offrir en victime pour le succès de cette noble entreprise».

6. On retrouve un unique système de pensée dans ces trois ouvrages. De l'un à l'autre, seule la disposition personnelle de l'auteur semble varier. L'enthousiasme de la Prière se transforme ainsi en amertume, voire en irritation, dans le Schisme. Son Christianisme intégral témoigne en revanche de l'apaisement de l'homme mûr face aux contingences du monde. 
vivante. De ce fait, elle est située dans l'espace et soumise à la loi de la croissance et du développement, comme tout organisme. Elle possède un centre capable de maintenir et d'énoncer l'una fides par un magistère infaillible. Toute coupure de ce magistère entraîne l'incapacité à participer au déploiement de la foi de façon certaine et unitaire. On assiste alors à la multiplication des opinions et à la dévitalisation des parties détachées du centre, qui se morcellent et qui s'effritent dans un mouvement de nécrose. Les chrétiens sincères et lucides doivent travailler à se dégager des forces centrifuges que constituent les aléas du monde, tels que le césaropapisme byzantin ou le phylétisme, et, par leur «accession ${ }^{7}$ spontanée à l'Église catholique romaine, lever les entraves qui freinent l'accroissement naturel de l'Église universelle.

Jugie déplore l'indifférence des «apologistes » catholiques de son temps pour les chrétiens orientaux. En effet, ceux-ci consument leurs énergies dans une vaine défense de l'Église face «aux rationalistes, aux protestants libéraux et aux modernistes ${ }^{8}$, alors qu'il serait beaucoup plus facile et efficace de gagner les Orientaux au catholicisme. Car les «Gréco-Russes » admettent d'emblée les principales vérités prônées par Rome. Il ne reste qu'à dissoudre l'obscurité doctrinale dans laquelle l'histoire les a placés, pour qu'ils saisissent rationnellement leurs erreurs et s'en défassent. Telle est la cause personnelle de Jugie, tel est également le but de toute son œuvre : faire admettre aux catholiques l'importance des chrétientés orientales ; faire comprendre aux orthodoxes leurs erreurs, par la force de la logique et une argumentation historique scientifiquement incontestable, et s'en faire des alliés. Sa perspective est donc fort éloignée d'une conception qui aurait pour objet le prosélytisme des masses ou la négociation avec telle ou telle Église. Elle est également étrangère à l'esprit de l'œcuménisme, mouvement perçu par lui comme chaotique ${ }^{9}$.

Jugie entend toucher la raison du chrétien, considéré individuellement. De ce fait, c'est avant tout aux intellectuels qu'il s'adresse, c'est d'eux qu'il espère une conversion qui - telle celle d'un John Henry Newman (18011890) venu de l'anglicanisme ${ }^{10}$ - donnera à l'Orient des théologiens capables de renouer avec le magistère infaillible:

L'important n'est-il pas de convaincre d'abord le dissident que son Église n'est pas la véritable? Une fois qu'il aura acquis cette conviction, il sera amené à chercher où se trouve le christianisme intégral. N'est-ce pas le chemin qu'ont suivi les grands convertis intellectuels de l'anglicanisme ou de l'orthodoxie orientale à l’Église romaine ${ }^{11}$ ?

7. Schisme, p. VII: «Nous disons accession et non retour, car pour retourner, il faut s'être éloigné par sa faute. Or, ce n'est nullement leur cas».

8. Christianisme intégral, p. XIX.

9. Schisme, p. 322.

10. Cf. M. Jugie, Une nouvelle dogmatique orthodoxe. Trois théologiens grecs en présence, EO 11, 1908, p. 264 : «Quand en aura-t-on fini avec cet esprit orthodoxe? Quand donc surgira le Newman de l'orthodoxie qui, se mettant loyalement et courageusement à l'étude de l'ancienne tradition, n'aura pas de peine à reconnaître que le grain de sénevé dont parle l'Évangile est planté sur le sol de l'Église romaine, où il n'a cessé de croître, même après que certains oiseaux se sont envolés de ses branches hospitalières?»

11. Christianisme intégral, p. 263. 
En particulier, il s'agira pour ces théologiens de revitaliser une foi figée au Concile de Nicée II (787) et, par référence à un centre redevenu fixe, lui permettre de se dégager de la variété des opinions, pour rejoindre le niveau de développement auquel est déjà parvenue l'Église catholique romaine :

Chez les Orientaux dissidents, plusieurs vérités définies par les conciles catholiques, après le septième, sont encore à l'état d'opinions théologiques librement débattues: en d'autres termes, les Orientaux dissidents retardent sur l'Église catholique pour ce qui regarde le développement dogmatique, ce développement s'étant arrêté chez eux à partir de leur rupture avec l'Église romaine ${ }^{12}$.

On comprend alors pourquoi Jugie scrute les écrits des théologiens orthodoxes de son époque. S'il écrit peu sur eux et n'engage pas directement le débat ${ }^{13}$, il guette dans leurs publications des indices présageant une levée du voile de l'obscurité doctrinale par l'usage de la raison et l'appréhension objective de l'histoire, ou plus simplement une ouverture personnelle à l'égard de l'enseignement de l'Église catholique. C'est probablement aussi ce qu'il espère de la part de Serge Boulgakov (1871-1944), quand il lui écrit pour la première fois en 1934.

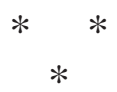

Nous publions ci-après deux lettres de Martin Jugie adressées à Serge Boulgakov et conservées dans les archives de l'Institut de théologie orthodoxe Saint-Serge à Paris, sous la cote XIII. 64. 6. Une pièce manque au dossier: la réponse de Boulgakov à la première lettre de Jugie; la seconde lettre y fait explicitement référence: «Je ne veux pas attendre plus longtemps à vous remercier de votre aimable lettre ». Mais nous n'avons trouvé cette réponse ni à la bibliothèque de l'IFEB, ni aux archives assomptionnistes à Rome ${ }^{14}$. Aucune copie n'est par ailleurs conservée à l'Institut Saint-Serge. Nous la considérons donc comme perdue, jusqu'à nouvel ordre.

Au printemps 1934, Jugie est à Lyon, où il enseigne à l'Institut catholique. Il s'y rend régulièrement depuis Rome, où il occupe la chaire de Théologie orientale à l'Athénée du Latran. En effet, cette discipline, qu'il a contribué à promouvoir par son œuvre, vient de s'imposer comme matière obligatoire dans l'enseignement supérieur catholique (1931), et ces deux institutions réclament sa présence de part et d'autre des Alpes. Il est également sur le point d'achever le cinquième et dernier volume de sa Theologia dogmatica, sur laquelle il travaille depuis dix ans. De son côté, Serge Boulgakov occupe depuis 1925 la position de Doyen de l'Institut Saint-Serge à Paris. Proche du métropolite Euloge Georgievsky (1864-1946), il est placé sous la juridiction du Patriarcat de Constantinople et participe activement au mouvement œcu-

12. Ibidem, p. XIV.

13. Cf. n. 10 et ses notices sur des théologiens orthodoxes du $20^{\mathrm{e}}$ siècle parus dans Catholicisme: liste dans Laurent, Euvre, p. 31-32.

14. Nos remerciements vont à Jean-Paul Périer-Muzet, A. A., archiviste de la Congrégation des Assomptionnistes, qui a bien voulu nous renseigner à ce propos. 
ménique. Avec le métropolite Germanos de Thyatire (1872-1951), il est l'une des figures de proue du groupe orthodoxe aux conférences de Foi et Constitution: Lausanne (1927), Édimbourg (1937) ${ }^{15}$. Il participe aussi à la création du Fellowship of St Alban and St Sergius (1928) qui rassemble orthodoxes et anglicans à Saint Albans, en Angleterre. Au moment où Jugie lui écrit, il vient de publier, en français ${ }^{16}$, L'Orthodoxie (Paris 1932) et, en russe, L’Agneau de Dieu (Paris 1933).

Le 2 mai 1934, Jugie, qui «vient de parcourir»L'Orthodoxie, prend contact avec Serge Boulgakov qu'il ne connaît pas personnellement et auquel il se présente. Bien qu'il semble n'avoir qu'un aperçu très partiel de son œuvre, il lui annonce son intention de rédiger «une étude d'ensemble» sur ses «doctrines et opinions dans la revue intitulée les Échos d'Orient, ou toute autre revue théologique catholique ». À cet effet, il lui demande de lui procurer ses «principales publications en matière théologique». Nous savons que pareille étude n'a jamais vu le jour ${ }^{17}$. Il est cependant légitime de rechercher aujourd'hui ce qui, de la seule lecture de L'Orthodoxie, a pu si « vivement intéresser» le théologien catholique, au point d'envisager ce travail.

Plusieurs positions de Boulgakov inspirées des Slavophiles du 19e siècle ont probablement suscité l'intérêt de Jugie qui, très attaché, comme nous l'avons vu, au caractère visible de l'Église, reste à l'affût du thème de l'Église invisible chez ses interlocuteurs ${ }^{18}$. En effet, dans L'Orthodoxie, la sobornost d'Alexis Khomiakov (1804-1860) est très présente, de même que des thèmes privilégiés par Vladimir Soloviev (1853-1900) : «L'Église est invisible ${ }^{19}$, «on ne peut définir les limites de l'Église ni dans l'espace ni dans le temps ${ }^{20}$, «la vie de l'Église est symbolique; c'est une vie mystérieuse cachée sous des signes visibles $»^{21}$.

15. Sur les activités de Serge Boulgakov à ces deux conférences, voir N. Zernov, The Eastern Churches and the Ecumenical Movement in the Twentieth Century, in R. Rouse, S. Ch. NeILl (éd.), A History of the Ecumenical Movement (1517-1948), I, Londres 1954, Genève $1986^{3}$, p. 654-660, et plus récemment Y. Provost, Le Patriarcat de Constantinople et les Églises orthodoxes à l'origine du mouvement acuménique (1902-1951). Le rôle particulier du métropolite Germanos de Thyatire, Mémoire dactylographié (EPHE-SR), Paris 2008, p. 3242.

16. Écrit en français et traduit en anglais la même année sous le titre The Orthodox Church, cet ouvrage, que certains jugent comme le meilleur de toute l'œuvre controversée de Boulgakov, ne fut traduit en russe qu'en 1964. La version russe fut traduite de nouveau en français par C. Andronikof et publiée en 1980.

17. Ni les indices des Échos d'Orient ni la bibliographie complète de Jugie établie en 1953 ne signalent une telle étude. On remarquera par ailleurs que la notice sur Serge Boulgakov donnée dans Catholicisme 2, 1949, col. 307-309, est due à I.-H. Dalmais, alors que celle sur Macaire Boulgakov, qui la précède aux col. 306-307, est écrite par M. Jugie.

18. Christianisme intégral, p. 148.

19. Orthodoxie, p. 5.

20. Ibidem, p. 9.

21. Ibidem, p. 10. 
Néanmoins, ce sont les affirmations de Boulgakov sur la primauté de Pierre, ainsi que sur la procession du Saint-Esprit, qui ont dû particulièrement retenir l'attention de Jugie. En effet, contrairement à certains théologiens orthodoxes qui nient la possibilité d'une primauté au sein de l'Église, Boulgakov soutient clairement celle de l'évêque de Rome:

[Le président] pour l'Église œcuménique, c'est naturellement le premier des patriarches, primus inter pares; les canons reconnaissaient comme tel l'évêque de Rome. Le monde orthodoxe continuerait à reconnaître au pape jusqu'à maintenant cette prérogative et sa qualité de premier patriarche œcuménique, s'il renonçait avec son Église locale aux prétentions vaticanes. L'Église orthodoxe n'a jamais nié cette primauté de la chaire romaine, confirmée par les canons des conciles œcuméniques ${ }^{22}$.

S'il rejette les deux thèses de l'infaillibilité et de la juridiction universelle du pape, énoncées par Vatican I, il n'en demeure pas moins qu'il se réfère à l'autorité de l'évêque de Rome comme à celle d'un centre fixe d'où procède le magistère de l'Église, par la décision des conciles. Le pape n'est donc pas infaillible, il représente cependant l'unité de l'Église et il en est la voix :

La primauté, dans le concile, appartient au pape [...]. C'est également au pape qu'appartiennent la proclamation des définitions des conciles, la présidence du synode œcuménique s'il venait à s'en former un, et pour ainsi dire, la représentation symbolique de l'unité de l'Église, qui naturellement tend à prendre forme en une seule personne ${ }^{23}$.

Quant au second point, celui de sa juridiction universelle, Boulgakov précise clairement $^{24}$ :

La primauté de juridiction n'a jamais appartenu dans l'Église universelle au premier patriarche, même au patriarche romain; il n'a eu qu'une primauté d'honneur (primus inter pares) ou d'autorité. Sans doute, en fait, l'autorité est quelquefois un pouvoir mais un pouvoir spirituel et non point canonique ${ }^{25}$.

En matière de doctrine trinitaire, c'est sans doute le point de vue développé par l'auteur de L'Orthodoxie sur le filioque qui a dû susciter le vif intérêt de Jugie. Boulgakov y voit, à l'origine, un théologouménon occidental, marquant ainsi une distance par rapport à ce que pouvait énoncer une théologie «photienne» et «figée». Dès la deuxième page de son ouvrage, il affirme:

22. Ibidem, p. 107-108.

23. Ibidem, p. 130.

24. On pourrait ainsi considérer Boulgakov comme un pionnier du dialogue théologique mené actuellement entre théologiens catholiques et orthodoxes, dans le cadre duquel le co-président orthodoxe J. Zizioulas, Service orthodoxe de Presse 298, 2005, p. 20, affirmait encore récemment: «Selon la Tradition, l'évêque de Rome est le premier évêque de l'Église entière. La difficulté avec la primauté de Pierre vient de ce qu'elle suppose une juridiction universelle par laquelle le pape peut interférer dans une Église locale. Mais si nous arrivions à trouver une conception de la primauté universelle du pape qui n'empiète pas sur la pleine nature de l'Église locale, nous pourrions l'accepter».

25. Orthodoxie, p. 129-130. 
L'amour de Dieu, l'amour du Père pour le Fils et celui du Fils pour le Père, n'est pas une simple qualité ou relation: il possède lui-même une vie personnelle, il est hypostatique. L'amour de Dieu est l'Esprit Saint, qui procède du Père au Fils et qui repose sur Lui. Le Fils n'existe pour le Père que dans l'Esprit Saint qui repose sur Lui: de même le Père manifeste son amour au Fils par l'Esprit Saint qui est l'unité de vie du Père et du Fils. Telle est la place du Saint-Esprit dans la Trinité $^{26}$.

Plus loin, il écrit à ce sujet:

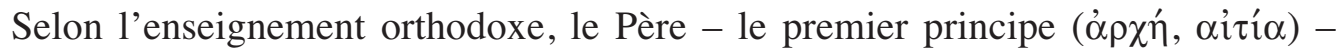
engendre le Fils avant tous les siècles et de lui procède le Saint-Esprit: c'est la monarchie divine dans la Sainte Trinité. C'est ici que se pose la question des rapports réciproques entre la seconde et la troisième hypostase. Selon la littérature patristique, l'Esprit Saint procède par ( $\delta$ เó) le Fils et repose sur lui ; telle est l'expression de leur lien quant à l'origine. En Occident cette corrélation, qui n'a jamais été claire dans l'Église, a été comprise comme une participation du Fils à la procession du Saint-Esprit. De là les discussions théologiques sur le filioque: causée d'abord par une différence d'opinions théologiques, la querelle se transforma ensuite en un désaccord dogmatique, parce que d'un theologoumenon occidental, on avait fait un dogme indestructible. L'expérience de la conférence de Bonn, entre Orthodoxes et Vieux catholiques, a montré qu'il est cependant possible d'aboutir à une compréhension théologique mutuelle sur ce point ${ }^{27}$.

Or, Jugie reviendra par deux fois sur la conception de Boulgakov relative à la procession du Saint-Esprit. Ainsi, dans son De processione Spiritus Sancti ex fontibus revelationis et secundum Orientales dissidentes (Rome 1936), il consacre trois pages à la doctrine trinitaire de celui-ci, en citant, en traduction latine, un long extrait de L'Agneau de Dieu et, en note, le passage de L'Orthodoxie reproduit ci-dessus. Les termes par lesquels il qualifie la doctrine trinitaire de Boulgakov sont les suivants :

Recentissime autem archipresbyter Sergius Bulgakov, Academiae theologicae russicae Parisiensis professor, novam de processione Spiritus Sancti theoriam protulit, quae ad explicationem augustinianam et thomisticam sub aliquo respectu appropinquat, nedum photianismo suffragetur. Ipsum Spiritum habet ut mutuum amorem Patris et Filii docetque Patrem et Filium unum effici in actu quo Spiritus procedit a Patre per Filium. Haud pauca vero profert, quae prorsus mira et inaudita sunt meraque phantasiae figmenta apparent ${ }^{28}$.

De même, il s'y réfère une nouvelle fois en 1947 :

Quant aux théologiens russes contemporains, qui, par l'examen de la tradition patristique, sont arrivés à la même conclusion que les anciens latinophrones et unionistes de la période byzantine, les principaux sont Jean de Kronstadt [...] et l'archiprêtre Serge Bulgakov, professeur de dogmatique à l'Institut de théologie russe de Paris [...]. Serge Bulgakov a une théologie tout à fait spéciale sur la Tri- 
nité, qui a été qualifiée d'hérétique dans certains milieux russes. En le rangeant parmi les théologiens favorables à la doctrine catholique, nous voulons simplement dire qu'il ne répugne pas aux formules catholiques: A Patre per Filium, a Patre Filioque ${ }^{29}$.

On remarquera cependant que les trois thèmes de l'Église invisible, de la primauté de Pierre et du filioque que nous avons présentés ici comme ayant pu susciter l'intérêt de Jugie pour l'œuvre du théologien russe et avoir été à l'origine de sa volonté d'établir un contact avec lui, ne sont pas évoqués directement dans les deux lettres. Ainsi, dans la première, il n'interroge son correspondant que sur un point précis qui semble lui poser problème, celui de «l'Eucharistie et de la transsubstantiation ${ }^{30}$.

Boulgakov estimait que transsubstantiatio et $\mu \varepsilon \tau \alpha \beta \lambda_{\eta} \eta \dot{~ s o n t ~ d e u x ~}$ concepts différents. Il affirme en effet dans L'Orthodoxie:

L'Église enseigne que le pain et le vin sont changés en Corps et en Sang du Christ donnés dans la sainte Cène. Mais l'Orthodoxie n'est pas d'accord avec la doctrine latine de la transsubstantiation où l'on distingue une substance qui change et des accidents qui ne changent pas $^{31}$.

Il ne faut pas oublier que Boulgakov a publié en 1930 un article en russe, intitulé «Le dogme eucharistique » ${ }^{32}$, auquel il renvoie dans L'Orthodoxie, mais auquel Jugie n'avait pas encore eu accès. Dans cet article, il critique la doctrine scolastique de la transsubstantiation qu'il considère être un point de vue absurde dérivé d'une évolution médiévale de la philosophie. Sur ce point, il reprend le point de vue d'Alexis Khomiakov qui considérait la doctrine de la transsubstantiation comme une approche de l'Eucharistie raisonnant avec les catégories de la chimie atomique ${ }^{33}$. Boulgakov y fait aussi une analogie entre le dogme de Chalcédoine sur la relation des natures humaine et divine du Christ, et le concept de $\mu \varepsilon \tau \alpha \beta \mathrm{h} \lambda \dot{n}$ à propos de la relation qui existe dans l'Eucharistie entre le pain et le vin d'une part, le Corps et le Sang du Christ d'autre part ${ }^{34}$. Mais il va peutêtre un peu trop loin lorsqu'il affirme que le corps du Christ qui est ressuscité et monté aux cieux est «parfaitement spirituel et à cause de cela absolument étranger à la nature du monde ou à la corporalité », qu'il est dans un état de «pure spiritualité » et qu'il a perdu sa «corporalité » ${ }^{35}$. Selon lui, le Christ serait apparu

29. Christianisme intégral, p. 156, avec la note 1.

30. Jugie publiera lui-même un ouvrage sur l'Eucharistie: De forma Eucharistiae, de epiclesibus eucharisticis, Rome 1943.

31. Orthodoxie, p. 160.

32. S. Boulgakov, Евхаристический догмат, Путь 20, 1930, p. 3-46; Путь 21, 1930, p. 3 33.

33. Cf. А. Кноміакоv, Несколько слов православного христианина о западных вероисповеданиях, Полное собрание сочинений, II, Moscou 1907, p. 131-132.

34. Ce point de vue de Boulgakov fut repris par le liturgiste russe N. UsPENSKY dans son article: Анафора. IV, Святоотеческое учение о Евхаристии и возникновение конфессиональных расхождений, Богословские Труды 13, 1975, p. 125-147. Il fait actuellement l'objet d'une polémique parmi les théologiens russes. Cf. M. Źeltov, Préface [Предисловие] à N. Uspensky, Византийская литургия. Историко-литургическое исследование, Moscou 2006, p. XXX-L.

35. Boulgakov, Евхаристический догмат, р. 39. 
à ses disciples après sa résurrection dans un corps «éthéré » ${ }^{36}$. Il en déduit que le corps du Christ ne peut être «physiquement » présent dans l'Eucharistie. On comprend la réaction de Jugie qui soutient n'avoir pas «saisi complètement la pensée » de son correspondant ${ }^{37}$.

La première lettre s'achève sur une question d'actualité relative au catéchisme de Khrapovitskii. On remarquera ici qu'Antoine Khrapovitskii (18631936) fut archevêque de Kharkov puis métropolite de Kiev avant de devenir le primat de l'Église russe hors-frontières, dont il fut l'un des fondateurs en 1923. Il publia à Sremski-Karlovci, en 1924, un Catéchisme chrétien orthodoxe dans lequel, voulant s'écarter de la doctrine scolastique anselmienne de la rédemption, il développa une théorie moraliste considérant l'agonie du Christ à Gethsémani comme base de l'œuvre rédemptrice. Cette théorie fit l'objet de critiques de la part de théologiens orthodoxes de l'époque, dont Georges Florovsky (1893-1979) ${ }^{38}$.

$$
*
$$

Encouragé par la réponse de Boulgakov et la réception de ses tirés à part, Jugie aborde de façon plus directe les sujets qui lui tiennent à cœur dans sa seconde lettre. Aussi n'hésite-t-il pas à exprimer clairement son attente d'une possible ouverture de son correspondant au catholicisme:

Il me semble que le vieux schisme byzantin [...] n'a plus, de nos jours, aucune raison d'être [...]. Croyez-vous qu'on ne pourrait s'entendre assez facilement entre Catholiques et Gréco-Russes? Il suffirait, à mon avis, d'un peu de bonne volonté et d'une sympathique étude réciproque, favorisée par des relations fraternelles [...]. Et le morcellement actuel de l'Orthodoxie ne peut-il suggérer d'utiles réflexions sur la nécessité d'un centre commun d'unité, de celui dont parlait l'évêque de Lyon, Saint Irénée?

Jugie est particulièrement intéressé par l'ecclésiologie de Boulgakov, notamment par son point de vue sur les profondes mutations subies par l'Église orthodoxe au début du $20^{\mathrm{e}}$ siècle : extension du nombre des autocéphalies à la suite de la création des nouveaux États baltes - dès 1923, le patriarcat œcuménique de Constantinople établit une Église estonienne autonome ${ }^{39}$-, Église orthodoxe en Russie sous le régime soviétique ou dans la diaspora.

36. Ibidem, p. 34 (voir la note).

37. Si l'étude annoncée par Jugie n'a pas vu le jour, la doctrine eucharistique de Boulgakov a fait l'objet d'un article critique de D. T. Strotmann, L'orthodoxie orientale dans le débat sur la transsubstantiation, Irénikon 32, 1959, p. 295-308. Sur cette question, voir aussi N. TzIRAKÈs, 'H

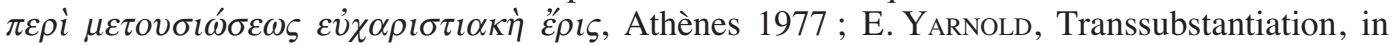
The Eucharist in Theology and Philosophy. Issues of Doctrinal History in East and West from the Patristic Age to the Reformation, éd. I. Perczel, R. Forrai, G. Gereby, Leuven 2005, p. 381394.

38. G. Florovsky, Les voies de la théologie russe, trad. fr. de J.-L. PALIERne, Lausanne 2001, p. 391.

39. L'Église d'Estonie s'autoproclama autocéphale en 1919 et fut reconnue comme Église autonome en 1923 par le Patriarcat œcuménique. L'Église de Lettonie s'autoproclama autocé- 
En effet, Boulgakov place au centre de sa réflexion la rupture produite par la révolution bolchevique sur cette symphonie byzantine qui a marqué, pendant près de dix-sept siècles, la relation de l'Église orthodoxe à l'État séculier et qui a contribué à la constitution d'une Église impériale. La chute de ce qui apparaissait comme le dernier Empire chrétien donne lieu à une situation nouvelle, et le nouveau mode de relation entre l'Église et l'État retient particulièrement l'attention des théologiens orthodoxes de Paris, à ce moment ${ }^{40}$. On peut ainsi lire dans L'Orthodoxie:

Sous ce rapport, l'Église est revenue à l'état des choses qui existait avant Constantin. Les souverains orthodoxes des États balkaniques ne peuvent être considérés comme chefs de royaumes orthodoxes, pareils à ceux de Byzance et de Russie. L'Église existe maintenant sans empereur, et sa situation charismatique, la plénitude de ses dons n'ont pourtant pas changé ${ }^{41}$.

Il faut dire que Boulgakov eut, dès 1932, le mérite de sortir du complexe d'impérialisme et de monarchisme qui modela pendant des siècles les rapports entre l'Église et l'État au sein de l'orthodoxie et dont souffrent, aujourd'hui encore, un certain nombre d'orthodoxes. Il opta personnellement pour « un nouvel aspect de la question des rapports entre l'Église et l'État, propre aux temps nouveaux», considérant que «si l'État peut être pénétré par l'esprit de l'Église, ce n'est pas extérieurement mais intérieurement, non d'en haut mais d'en bas ${ }^{42}$, et rappelant que la thèse du pouvoir monarchique «n'est jamais devenue un dogme et ne pouvait le devenir ${ }^{43}$. Il écrit ainsi :

Il n'y a pas de lien dogmatique entre l'Orthodoxie et un système politique déterminé. L'Orthodoxie est libre et ne doit pas servir un régime politique. Elle possède un idéal religieux, et non politique, de la sanctification du pouvoir; et ce n'est pas l'idéal des deux glaives ou celui d'un État ecclésiastique tel que la monarchie pontificale, à laquelle le catholicisme ne veut renoncer. L'Orthodoxie n'admet ni papo-césarisme, ni césaro-papisme ${ }^{44}$.

Mais la question cruciale pour Jugie, celle pour laquelle il «souhaite rencontrer» son correspondant, est sans doute celle de l'unité de l'Église, sujet du quatrième chapitre de L'Orthodoxie. Ce théologien, qui raisonne en termes de «centre» et de «parties séparées » voire «morcelées », se préoccupe de la

phale en 1926 et fut reconnue comme Église autonome en 1936 par le Patriarcat œcuménique. La situation de l'Église de Lituanie fut quant à elle un peu différente, l'autocéphalie lui ayant été accordée par un décret du synode du Patriarcat de Moscou en 1928.

40. Voir, par exemple, C. Bezobrazov, Царство Кесаря перед судом Нового Завета, Да приидет Царствие Твое, Paris 2003, p. 157-187; А. KARTAŠOv, Церковь и государство, Varsovie 1937; IDEM, Достижима ли христианская государственность?, Церковный вестник Западно-Европейского Православного Русского Экзархата 21, 1949, р. 32-33. Voir, à ce sujet, R. TODER, Космологические споры в трудах профессоров СвятоСергиевского Православного Богословского Института в Париже (историко-богословский обзор), Труди Київської Духовної Академії 8, 2008, p. 67-80.

41. Orthodoxie, p. 224.

42. Ibidem, p. 225.

43. Ibidem, p. 226.

44. Ibidem, p. 229. 
définition que Boulgakov donne du système de l'autocéphalie. En effet, selon ce dernier, «l'union dans la pluralité » ou «l'unité multiple ${ }^{45}$, qui laisse intacte la «diversité historique » concrète des groupes ${ }^{46}$ et permet l'apparition de juridictions parallèles dans la diaspora, peut assurer la cohésion de l'Église une. D'ailleurs, cette approche orthodoxe correspondrait davantage à la modernité :

L'organisation décentralisée de l'Orthodoxie, cette coexistence d'Églises nationales, autonomes mais unies, correspond beaucoup plus à l'esprit contemporain que le centralisme de Rome ${ }^{47}$.

Considérant que si «les Églises nationales autocéphales conservent leur caractère historique concret, elles peuvent suivre leur ligne propre», Boulgakov est toutefois conscient des faiblesses de ce système. Il affirme en effet :

Certainement, cette variété entraîne quelques conséquences fâcheuses, quelques différences trop accentuées, mais ces particularités n'abolissent pas l'unité et ne peuvent être supprimées.

Et, quelques lignes plus bas, il devient encore plus critique, allant même jusqu'à se contredire :

La pluralité des Églises autocéphales apporte dans la vie de l'Église plus d'inégalités et de dissonances: elle entraîne un provincialisme inévitable qui combat d'ailleurs, de nos jours, le processus de nivellement du monde civilisé ${ }^{48}$.

Or pareilles positions allaient bien dans le sens des attentes de Jugie.

Une seconde question d'importance apparaît en filigrane dans la seconde lettre. Il s'agit de la polémique autour de L'Agneau de Dieu. Même si la controverse sophiologique n'est pas mentionnée par Jugie, il ne faut pas oublier que les deux lettres rédigées en mai 1934 sont écrites en plein milieu du débat théologique qui culminera sur la double condamnation de Boulgakov par le patriarcat de Moscou et le synode de Carlovitz de l'Église russe hors-frontières en $1935^{49}$. Sans sous-estimer l'importance de la controverse théologique sur la sophiologie, on remarquera ici qu'elle a servi de prétexte dans les débats autour des clivages ecclésiastiques qui ont divisé la diaspora orthodoxe russe au $20^{\mathrm{e}}$ siècle. Nous l'avons dit plus haut, Boulgakov était proche du métropolite Euloge Georgievsky ${ }^{50}$, qui l'avait protégé lors de la controverse et qui avait placé son diocèse - généralement désigné par le

45. Ibidem, p. 126, 131.

46. Ibidem, p. 130.

47. Ibidem, p. 132.

48. Ibidem, p. 131.

49. Voir à ce sujet C. Lialine, Le débat sophiologique, Irénikon 13, 1936, p. 168-205, 704705.

50. Voir l'autobiographie d'E. Georgievsky, Le chemin de ma vie, Paris 2005 ; N. Ross, La rupture entre le métropolite Euloge et le métropolite Serge, vue de la rue Daru (1927-1931), Istina 53, 2008, p. 76-100. 
lieu de son siège comme «la rue Daru»-sous la juridiction du patriarcat œcuménique de Constantinople. Lancées par les deux autres branches ecclésiales de la diaspora orthodoxe russe en Europe occidentale - l'Église russe hors-frontières et l'Exarchat du Patriarcat de Moscou -, les attaques contre la théologie de Boulgakov, jugée hérétique, servirent en quelque sorte de prétexte pour discréditer l'Exarchat de «la rue Daru».

Même s'il n'avait pas encore lu L'Agneau de Dieu, dont il demande dans sa lettre un exemplaire, Jugie ne pouvait ignorer la controverse dont ce livre faisait l'objet. À Rome, où il résidait habituellement, Th. Spáčil préparait déjà, sans aucun doute, son long article sur cet ouvrage - un article que Jugie cite en 1936 -, et il est fort probable que les deux savants en aient discuté ${ }^{51}$.

Si le théologien catholique prend bien garde de critiquer son correspondant sur ces questions sensibles qui touchent à la doctrine, il ne peut pas s'empêcher de se livrer à l'étonnement, voire à une pointe d'ironie, sur deux autres questions: celle du rapprochement de Boulgakov avec les anglicans, qu'il évoque indirectement, parlant plutôt de «Russes de l'émigration » qui «penchent pour l'Église anglicane»; celle de sa distance doctrinale avec son «homonyme Macaire Boulgakov», dont les vues «diffèrent assez sensiblement» des siennes. Les relations entre orthodoxes et anglicans auxquelles il est fait référence sont sans doute liées à la fondation du Fellowship of St Alban and St Sergius sous l'impulsion de Nicolas Zernov (1898-1980), qui avait éprouvé, dès 1923, lors d'une réunion de la Fédération universelle des associations chrétiennes d'étudiants, une communion dans la même foi chrétienne avec les anglicans. Placée sous le patronage de saint Alban, premier martyr chrétien d'Angleterre, et de saint Serge de Radonège, illustre moine russe, cette association regroupait, côté anglican, les héritiers du Mouvement d'Oxford et, côté orthodoxe, les professeurs et les étudiants de 1'Institut SaintSerge. Or Boulgakov y était une personnalité dominante ${ }^{52}$. En 1932, il était même allé jusqu'à prôner l'intercommunion entre les anglicans et les orthodoxes qui seraient parvenus à un accord doctrinal ${ }^{53}$.

Quant à la pointe d'ironie relative à la différence de vues des deux Boulgakov - Macaire que Jugie connaissait bien ${ }^{54}$ et Serge qu'il commençait à découvrir -, on peut dire seulement qu'elle exprime très laconiquement l'une des thèses fondamentales de l'auteur de la Theologia dogmatica. Comme il y eut, pour lui, «autant d'orthodoxies que d'orthodoxes » ${ }^{55}$, son but 92.

51. Th. SpÁčll, Nova opinio auctoris orthodoxi de unione hypostatica, $O C P 1,1935$, p. 53-

52. N. Zernov, Fellowship of St. Alban and St. Sergius. A Historical Memoir, Oxford 1979; E. Behr-Sigel, Un moine de l'Église d'Orient, Paris 1993, p. 353-370.

53. Zernov, Eastern Churches, cité n. 15, p. 663.

54. Jugie est l'auteur de deux notices sur Macaire Boulgakov (1816-1882) dans Catholicisme (cf. n. 17) et dans le DTC 9, 1927, col.1443-1444. Il le cite d'ailleurs fréquemment dans sa Theologia dogmatica (voir les tables des vol. I-IV).

55. Christianisme intégral, p. 166 et p. 154. Jugie présente ce point de vue dès 1908, dans EO 11, p. 146: «Si en Occident, rien n'est plus banal que l'apparition d'un nouveau manuel de théologie, en Orient, surtout en Grèce, c'est un phénomène rare. Aussi les amateurs de théologie 
fut, avant tout, de «faire l'histoire des variations de l'Église gréco-russe des origines à nos jours ${ }^{56}$, et cela pour en montrer l'écart par rapport à l'una fides soutenue par le magistère infaillible.

Nous ignorons si cette correspondance eut une suite, ou si les deux hommes se rencontrèrent. De nouveaux documents permettront peut-être de répondre à ces questions à l'avenir. Mais il est certain que chacun suivit, après 1934, sa propre voie de chrétien: Serge Boulgakov, par son attachement au développement de l'Institut Saint-Serge, dont il fut le Doyen jusqu'à sa mort, en 1944, ainsi que par sa participation de plus en plus active au mouvement œcuménique; Martin Jugie, par ses recherches historico-doctrinales conduites à l'ombre du Vatican, mais également par son œuvre mariologique, qui lui valut de voir, de son vivant, l'Assomption de Marie promue au rang de dogme énoncé ex cathedra par l'évêque de Rome (1950) ${ }^{57}$. Nombre de thèmes soulevés dans ces deux lettres et évoqués ici ont quant à eux connu leurs propres développements et restent aujourd'hui encore d'actualité ${ }^{58}$.

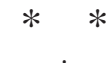

orthodoxe sont-ils à l'affût de ces sortes de publications. Ils espèrent toujours y trouver quelque chose de neuf. L'expérience leur a appris que, sur un grand nombre de points de la dernière importance, il y a, au sein de l'orthodoxie, quot capita tot sensus».

56. LaURent, Euvre, p. 12.

57. Ibidem, p. 15. Il s'agit de son œuvre majeure, La mort et l'Assomption de la Sainte Vierge. Étude historico-doctrinale (Studi e Testi 114), Vatican 1944.

58. On en retiendra deux ici : -1 . la réflexion sur les dogmes de l'infaillibilité du pape et de sa juridiction universelle promulgués par Vatican I, lesquels, confrontés à l'ecclésiologie de Vatican II, constituent aujourd'hui un problème majeur pour le dialogue œcuménique. Voir J. RATZINGER, Les principes de la théologie catholique. Esquisse et matériaux, Paris 1985, p. 221-223; W. KASPER, Principes herméneutiques pour la relecture des dogmes de Vatican I. À la veille de la reprise du dialogue catholique-orthodoxe, Istina 50, 2005, p. 341-352; H. Legrand, Pour une approche procédurale de la primauté romaine, Istina 53, 2008, p. 23-46. 2. la question du traitement du filioque, la vision de Boulgakov sur l'Esprit Saint qui «procède $\operatorname{par}(\delta i \alpha ́)$ le Fils et repose sur lui» ayant inspiré le débat théologique et œcuménique du $20^{\mathrm{e}}$ siècle. Celui-ci aboutit en 2003 à la Déclaration commune sur le filioque de la Commission théologique orthodoxe-catholique d'Amérique du Nord: Le filioque, une question qui divise l’Église ?, Irénikon 77, 2004, p. 69-100. 
Archives de 1'Institut de Théologie Orthodoxe Saint-Serge Fonds S. Boulgakov, n XIII. 64.6

\section{Première LetTre}

Lyon-V, 2, Avenue Debrousse, le 2 mai 1934

\section{Monsieur l'Archiprêtre,}

Je viens de parcourir l'ouvrage que vous avez publié chez Alcan, intitulé «L'Orthodoxie», et il m'a vivement intéressé. Malheureusement, sur certains points, par exemple sur l'Eucharistie et la transsubstantiation, je n'ai pas saisi complètement votre pensée, n'ayant pas les autres ouvrages et articles que vous avez publiés en russe et autres langues en ces dernières années et auxquels vous renvoyez le lecteur. Je vous serais bien obligé, si vous pouviez me procurer vos principales publications en matière théologique pour une étude d'ensemble que je me propose d'écrire sur vos doctrines et opinions dans la revue intitulée les «Échos d'Orient», ou toute autre revue théologique catholique. Depuis plus de trente ans, j'ai fait ma spécialité de l'étude de la théologie gréco-russe et j'ai publié en ces dernières années chez Letouzey (87, Boulevard Raspail, Paris) quatre volumes en latin sur la matière. En fait de théologie russe, je n'ai pas dépassé l'année 1917, sauf pour ce qui regarde le Catéchisme publié à Carlovitz, en 1924, par le métropolite Antoine Khrapovitskii. J'entends dire qu'il l'aurait retiré devant les attaques qu'il a suscitées dans les milieux orthodoxes et en particulier parmi les Russes émigrés. Il est entendu que je vous adresserai au moins deux exemplaires, et même plus, si vous le désirez, du travail que je me propose d'écrire sur votre doctrine théologique.

Veuillez agréer, Monsieur l'Archiprêtre, l'expression de ma haute considération et de ma fraternelle charité in Christo.

P. Martin Jugie, des Augustins de l'Assomption,

Professeur de théologie orientale à l'Athénée du Latran, à Rome, et à l'Institut catholique de Lyon,

Membre correspondant de l'ancien Institut archéologique russe de Constantinople

2, Avenue Debrousse, Lyon-V.

\section{DEUXIÈME LETTRE}

Lyon-V, 2, Avenue Debrousse, le 10 mai 1934

Monsieur l'Archiprêtre,

Je ne veux pas attendre plus longtemps à vous remercier et de votre aimable lettre et des tirages à part que vous avez bien voulu m'envoyer ainsi que du catalogue de vos œuvres. Si YMCA-Press ne peut m'envoyer un exemplaire de presse de votre grand ouvrage L'Agneau de Dieu, je l'achèterai. Ce qui m'intéresse surtout, ce sont vos vues sur l'ecclésiologie. Je souhaite qu'une occasion nous permette de nous rencontrer. J'aurais bien des questions à vous poser, des éclaircissements à demander sur la situation des Églises russes en Russie et hors de Russie. Si vous aviez l'occasion de passer à Lyon avant le mois de juillet, ne manquez pas de me prévenir pour que je puisse vous faire une visite. 
Je cherche des renseignements sur les nouvelles Églises autocéphales d'Esthonie, de Lettonie et de Lithuanie. Si vous connaissiez quelque document écrit qui parle de ces nouvelles autocéphalies, je vous serais très reconnaissant de me le signaler.

Je trouve que vos vues, sur certains points de doctrine, diffèrent assez sensiblement de celles de votre homonyme Macaire Boulgakov. Il me semble que le vieux schisme byzantin, dû surtout au mépris des Byzantins policés pour les Barbares d'Occident, n'a plus, de nos jours, aucune raison d'être. Les querelles théologiques, presque toujours puériles, ne furent que des prétextes. Croyez-vous qu'on ne pourrait pas s'entendre assez facilement entre Catholiques et Gréco-Russes? Il y suffirait, à mon avis, d'un peu de bonne volonté et d'une sympathique étude réciproque, favorisée par des relations fraternelles. Les Russes de l'émigration sont bien placés pour étudier de près l'Église romaine, qu'on défigurait à plaisir en Russie, avant la guerre. Je sais que plusieurs d'entre eux penchent pour l'Église anglicane, et cela me surprend beaucoup, à moins qu'il ne s'agisse de la High Church. Comment une Église, qui n'a commencé qu'au XVI ${ }^{\mathrm{e}}$ siècle et dont le Prayer Book contient de notoires hérésies pourrait-elle être l'antique Catholica des Pères? Et le morcellement actuel de l'Orthodoxie ne peut-il suggérer d'utiles réflexions sur la nécessité d'un centre commun d'unité, de celui dont parlait l'évêque de Lyon, Saint Irénée?

Il est bien entendu que je vous adresserai quelques tirages à part de l'étude que je me propose d'écrire sur votre théologie. Je m'efforcerai de n'employer aucune expression qui puisse être blessante pour vous et offenser la charité chrétienne. Si j'attaque les doctrines, mon intention est toujours de ne pas offenser les personnes.

Veuillez agréer, Monsieur l'Archiprêtre, avec l'expression renouvelée de ma reconnaissance, mes sentiments respectueux et fraternels in Christo.

Martin Jugie

Job GETCHA, ITO Saint-Serge

Vassa Kontouma-Conticello, EPHE et IFEB 